\title{
How Events Affect Trust: A Baseline Information Processing Model with Three Extensions
}

\author{
D. Harrison McKnight, Peng Liu, and Brian T. Pentland \\ Michigan State University \\ \{mcknight, liup, pentland\} @bus.msu.edu
}

\begin{abstract}
This article addresses how trust changes over time. We introduce a social psychology-based Information Processing Model (IPM) that explains how trust changes over time based on three cognitive mechanisms: attention, attribution, and judgment. This model is contrasted with the traditional incremental progression model of trust change. We also explain three extensions of the model. These models are then simulated and the results suggest that incremental progression may be inconsistent with established psychological theory.
\end{abstract}

Keywords: Trust, attention, attribution, threshold, risk, illusion, change.

\section{Introduction}

We define trust as the extent to which one is willing to depend on, or become vulnerable to another with a feeling of security despite situational risk. Based on socialpsychological theory, this paper introduces a baseline information processing model (IPM) of how trust changes in response to external events. We then extend the baseline model in three ways and do simulations. The simulations suggest trust changes much less frequently, but in larger chunks, than in an incremental change model. We employ a process theory lens. Process theories specify which temporally-ordered occurrences are necessary for other occurrences to happen. They address how a phenomenon changes over time. Current trust change models offer a simple view of how trust changes, such as growth in small incremental steps as parties interact (e.g., [1]).

These "incremental step-by-step" trust progression models indicate interactional events lead to changes in trust levels. This helps specify the general trust change dynamic. Each positive event increases trust. However, they address the behavioral level and do not connect to the underlying cognitive processes by which humans process events. These models treat actors' detailed cognitive processes as a black box.

By contrast, Newell [9] presents a powerful example of detail-level theorizing. Newell argues that our knowledge at the biological level (e.g., neuron firing rates) should be predictive of phenomena observed at the cognitive or behavioral level (e.g., reaction time). The more detailed biological level helps explain how the cognitive level phenomenon works. Similarly, we believe that processes at the cognitive level should help explain how the process of trust changes work at a behavioral level. One 
reason this approach is needed is that empirical work often does not support the step-by-step trust progression models. For example, Jarvenpaa and colleagues [3] find almost no difference in virtual class team trust levels between T1 and T2. Rather, high trust teams maintained high trust and low trust teams maintained low trust. Incremental trust progression theory would predict small changes would occur based on experience. The lack of support suggests better theories of trust progression are needed.

Our IPM model builds on cognitive social psychology research because trust between people is primarily a social, mental construct [6] embodying perceptions. We focus on one person's trust perceptions with respect to one other party, either another person or a technology partner. Some of what we argue may not apply to a technology trusting a technology. The model is especially appropriate for soft trust situations or for studying reputation effects. The IPM contributes to theory by ordering the cognitive events needed to produce trust changes, an aspect of process theorizing. While the steps occur in a set temporal order, the outcome of the steps is not deterministic.

First, model action initiates when an event takes place that may have trust ramifications (Figure 1, Node 1.). The event is typically an interaction with the trustee. The model proceeds clockwise. Node 2. on Figure 1 (attention module) refers to whether the trusting person notices or pays attention to the event, which is important to trust (e.g., [7]). If the event is not noticed (2. "No" path), no subsequent steps are completed, trust stays the same, and the process awaits the next event. Attention necessarily precedes inference/attribution: "If events are noticed, people make sense of them; and if events are not noticed, they are not available for sensemaking" [4: 58-59].

Scholars posit that trust is updated through a mental attribution process [5]. After attribution, the trusting person assesses whether the attributed contradiction is serious enough to exceed the threshold cost of updating the trust level (node 4.). If not, the trust level is not updated and processing awaits the next event. If so, the update node is initiated, which increases / decreases the trust level. The person then brings a new level of trust to the perceptual system that encounters the next event. Not only is the trust level updated, but the likelihood of attending to and attributing about an event are also updated. We now briefly justify the model.

\section{The Baseline Information Processing Model}

By attention, we mean the person expends cognitive effort on the event instead of ignoring it. The mind only consciously processes a small percentage of stimuli. People often ignore information that does not match what they believe. An event passes through the trustor's perceptual / memory module before they pay attention to it (Figure 1). Thus attention is a necessary mental process that occurs before a trust change takes place. Although attention is necessary, it is not sufficient to change trust. Instead, the trustor must go through attribution and judgment subprocesses first [5].

Assumption 1: Trustor attention to a behavioral event is a necessary but not sufficient condition for the change in a trustor's level of trust.

Attribution means how a person makes sense of an event. One ascribes underlying qualities or enduring motivations to the other based on observable evidence. Because 


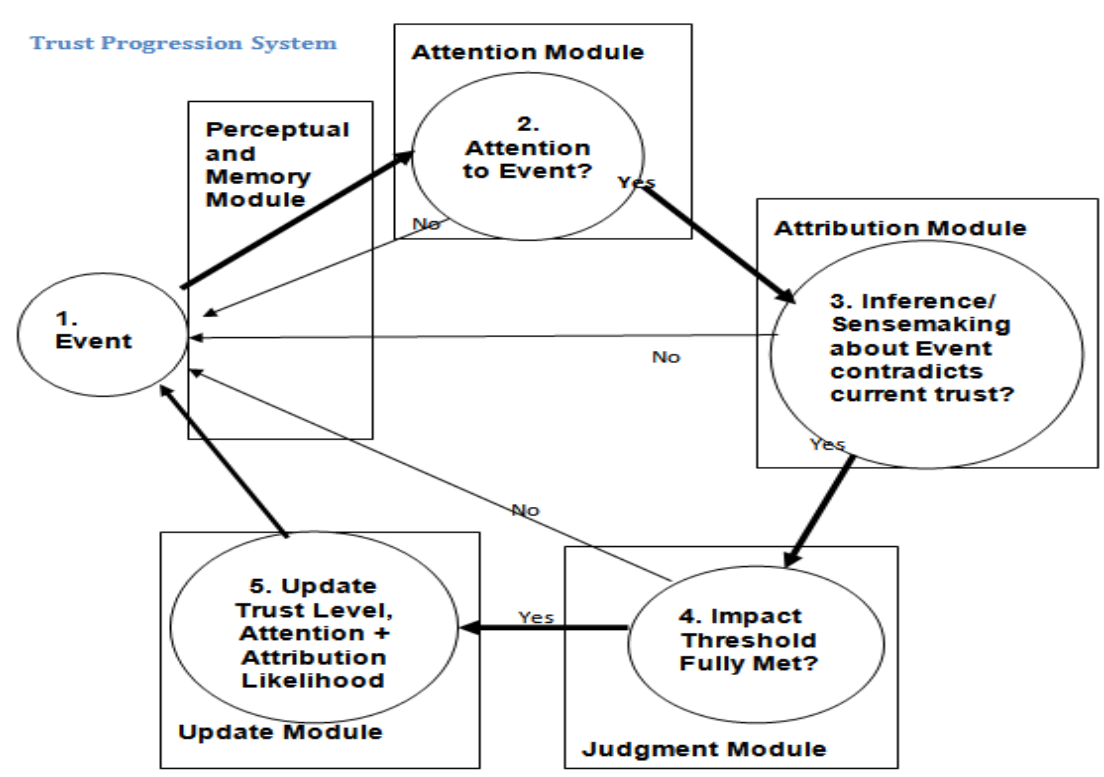

Fig. 1. Trust Development: A Baseline Information Processing Model (IPM)

attributions are subjective and affected by mood, their outcomes are hard to predict. Only when the cause of an event is decided can trust be updated, and only when the event's attribution contradicts one's existing trust perceptions is an update made. A trustor must perceive a clear contradiction between the existing trust level and eventimplied trustworthiness before trust is updated. Even after a contradictory attribution takes place, a threshold judgment must still take place. Hence, attribution is necessary but not sufficient for a change in trust.

Assumption 2: Trustor attribution that a behavioral event contradicts current trust levels is a necessary but not sufficient condition for the change in the level of trust.

After making an attribution, a trustor judges whether it meets the threshold for revising trust levels. This is termed a judgment module because it compares the reasons or benefits for updating trust with the costs of updating trust. Judgment is defined as making a size comparison between two objects, which we argue are the perceived costs and benefits of a trust level change. Changing trust is cognitively expensive because trust is a central, highly connected relationship concept. If you alter trust, you have to change a number of other beliefs, such as liking and respect of O. It costs less to maintain existing beliefs and consider the event a quirk. Holmes [2] and Luhmann [5] discuss how trust changes involve thresholds.

Assumption 3: Exceeding a cost-versus-benefit threshold for changing trust is a necessary condition for the change in a trustor's level of trust.

Once the threshold is exceeded, the trust level is updated by some amount. The likelihood of attending to and attributing about the next event will also be affected. If trust goes down, one will be more likely to notice and attribute about the next event. 
The IPM shows why trust changes are hard to predict [5]. Each of three sequential, probabilistic, inter-related process mechanisms (attention, attribution, threshold judgment) must operate consecutively for trust to change. The IPM also suggests that trust trends will tend to be "sticky." Once a trust level is firmly fixed, it may be hard to change. The incremental progression model suggests that small, frequent changes occur. Instead, the IPM suggests changes will be relatively infrequent and large.

\section{Three Model Extensions—Risk, Negative Change, Illusion}

Perceived situational risk is important to trust attribution. Situational risk means the likelihood that negative consequences will occur in the context. The higher the perceived situational risk, the more one will attend to an event and make attributions about it. Low risk situations do not get one's attention like high risk ones do. Similarly, attribution takes mental energy that will not be expended unless one thinks the risk justifies it. Thus, low risk situations will produce less effortful attribution.

Assumption 4: Higher perceived situational risk will increase the likelihood of both attention and attribution to events.

Will trust decreases be greater in magnitude than trust increases? The literature suggests the answer is "yes." Trust is easier to destroy than build, and cognitive research suggests negative trust-related events are easier to remember and have a greater relative effect than do positive events.

Assumption 5: Negative changes to trust will be significantly greater in magnitude than will positive changes in trust.

In committed romantic relationships people idealize their partner and use that idealized perception to dispel the effects of negative events [7]. This may also occur in business relationships. Illusion means the extent to which a partner is more optimistic about their relationship's future than rationally merited. When one has positive illusions, a set of positive/negative events will continue those illusions. Such a pattern provides equivocal evidence about $\mathrm{O}$. However, when negative evidence is consistent across events, the evidence will be considered less equivocal. Then the illusionary "bubble" will burst and the pattern will stop until a consistent positive pattern occurs.

Assumption 6: Illusionary partners will reinterpret negative events as positive events. An accumulation of negative events will remove this reinterpretation effect, which will continue until an accumulation of consistently positive events reinstates it.

\section{$4 \quad$ Method}

\subsection{Baseline Model}

We simulate the above using MATLAB 7.11, as follows.

Events (E). A positive value means a good event and a negative value a bad event. Event values follow a normal distribution with zero as mean and one as standard deviation $(E \sim N(0,1))$. The total number of events is $N=1000$. 
Memory (M). In this model, we assume $\mathrm{M}=10$ which means a person remembers the most recent past ten events. When a new event is remembered, the oldest event is "forgotten." Memory will decay over time, as the value of events in the memory decreases by $10 \%$ as every new event happens $\left(E_{T}=E_{T-1} \times 0.9\right)$.

Attention (A). Attention is the probability that one notices an event. Assuming a low baseline risk level, we set the probability at $30 \%$ chance that one will attend to an event. As trust increases, attention decreases (in proportion as trust changes), because people who feel secure pay less attention. When trust decreases, attention increases.

Attribution (R). The probability of full attribution about the event is Attribution (R). Because of the complex issues for Assumption 2 above, we set the initial probability of attribution at 0.3. An increase in trust leads to a lower probability of attribution about the next event. A decrease in trust leads to a higher probability.

Threshold (Th). To model this, we put a threshold of 0.5 for the baseline model. If the absolute value of the sum of the event values in memory is greater than the threshold, trust will be updated; otherwise trust will remain the same.

Trust (T). This model keeps track of the trust level (T) of a person and how it changes when a person experiences a stream of random events. Trust ranges from 0.0 to 1.0. Initially, we set the trust level to 0.5 . This reflects the assumption that people give each other the benefit of the doubt when they first start to deal with each other [5]. When trust is being updated, the change of trust is one half the difference between the absolute value of the sum of the event values in memory and the threshold value. For example, if the sum is positive and $20 \%$ higher than the threshold, then trust will increase by $10 \%$; and attention and attribution will decrease by $10 \%$. If the sum is negative and the absolute value of the sum is $15 \%$ higher than the threshold, then trust will decrease by $7.5 \%$; and attention and attribution will increase by $7.5 \%$.

\subsection{Three Extensions That Affect the Baseline Model}

\subsubsection{Situational Risk Model}

There are high risk, medium risk and low risk situations $(0.9,0.6$, and 0.3$)$. The baseline model has low risk, with initial attention and attribution at 0.3 , and memory effect at 10. The starting values of attention (A) and attribution (R) are equal to the value of the event risk (ER). When risk is high, there is no change in the attention and attribution; when risk is medium, the change of attention and attribution is $50 \%$ of what the baseline model says they should change; when risk is low, the change of attention and attribution is governed by the baseline model. Higher risk has a long memory effect ( 20 events recalled), medium risk has a medium memory effect (15 events), and low risk has a short memory effect (10).

\subsubsection{Negativity Asymmetry Model}

In the stronger negativity effect model, a negative event has twice the effect on trust as a positive event. So when a negative effect triggers the trust change, trust will change double what it would change in the baseline model; the baseline model governs the effects of a positive event. 


\subsubsection{Illusion Model}

When the illusion effect is on (including initially), trust will keep increasing by taking the absolute value of the difference between the sum of the entire event values in memory and the threshold, even if a negative event triggers the trust change. But when there are three negative events in a row, the illusion effect is turned off, and the model becomes the baseline model and trust goes either up or down. If three positive events occur in a row, this turns back on the illusion effect.

\section{$5 \quad$ Results}

The following tables display simulation results. The incremental progression model simulates trust changing after each event, with the change magnitude equaling the event size $(\mathrm{E} \sim \mathrm{N}(0,0.1))$. Table 1 also depicts medians of the magnitude, frequency, and trend of the trust level across 1000 events. Magnitude means the average size of the change in trust level for an event. Table 1 shows that the median baseline model magnitude of change is over twice that of the incremental progression model. Frequency means the number of changes in trust that have occurred divided by the number of events. Frequency of trust change is significantly lower for the baseline model and its extensions than for the incremental progression model. Trend means the average number of times trust changes in the same direction (e.g., increase) before it starts changing in the other direction (e.g., decrease). Note that the direction changes often for the incremental progression model, yielding a low trend figure (1.99), while in the other models trust progresses longer without changing direction.

Table 2 shows the first 200 events of three randomly selected simulations to illustrate the shape of the trust progression curve. The incremental progression model shows that trust changes often in small random increments. The baseline model shows a more infrequent change pattern with higher magnitude of the average trust change. The negativity asymmetry shows a similar trend, but with a decreasing direction. The medium and high situational risk models have even wider swings, both up and down.

Table 1. Medians for Simulated Model Instances

\begin{tabular}{|lccc|}
\hline \multicolumn{1}{|c}{$\mathrm{n}=1000$} & Magnitude & Frequency & Trend \\
\hline Incremental Progression Model & 0.023 & 0.835 & 1.998 \\
\hline Baseline Model (Low Risk) & 0.049 & 0.097 & 4.280 \\
\hline Negativity Asymmetry Model & 0.044 & 0.124 & 5.000 \\
\hline Medium Risk Model & 0.055 & 0.146 & 4.567 \\
\hline High Risk Model & 0.056 & 0.526 & 7.725 \\
\hline Illusion Model & 0.054 & 0.096 & 5.875 \\
\hline
\end{tabular}

\section{Discussion}

The simulation results are quite striking. First, as shown in Table 1, the magnitude of trust changes is much higher in every version of the IPM model than for the 
conventional incremental change model. The difference is particularly pronounced in high risk situations, where trust is most important. Qualitatively, this is a good fit with empirical results, which show that trust levels can make wide, sudden swings.

Table 2. Random Sample Displays of Trust Progression under Different Assumptions

\begin{tabular}{|c|c|c|c|}
\hline 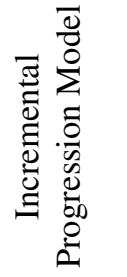 & 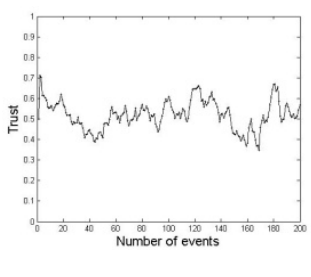 & Nons & 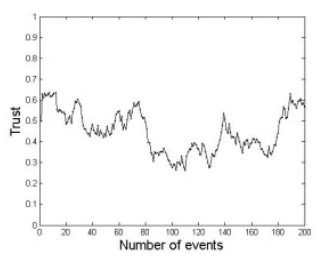 \\
\hline 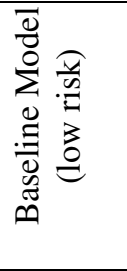 & (1) & (i) & (1) \\
\hline 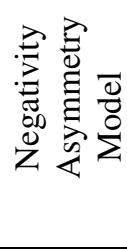 & (1) & 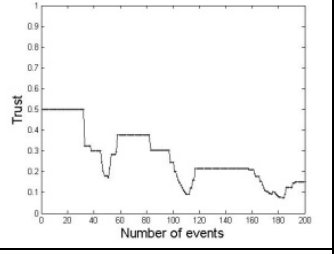 & (1) \\
\hline 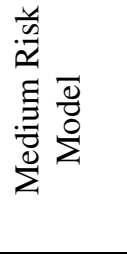 & 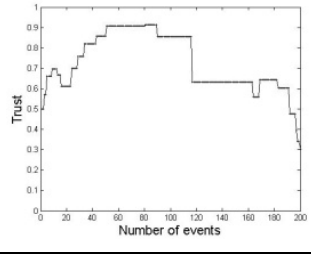 & (in) & 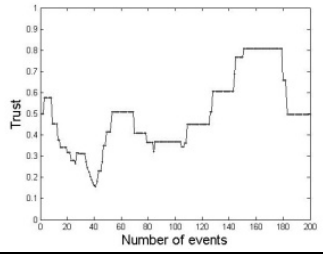 \\
\hline 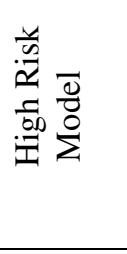 & (1) & 我 & . \\
\hline $\begin{array}{l}\overline{0} \\
\frac{0}{0} \\
\sum_{0} \\
0 \\
\frac{0}{0} \\
\equiv\end{array}$ & 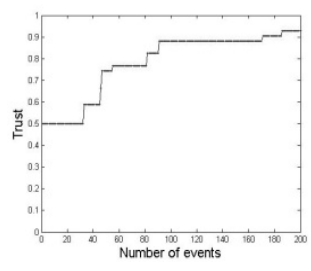 & 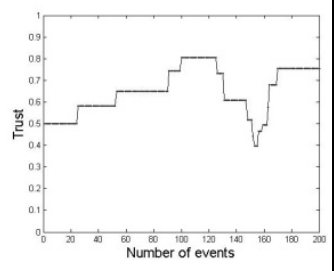 & 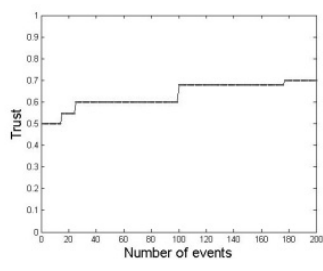 \\
\hline
\end{tabular}


Second, as shown in Table 1, the frequency of change is much lower in every version of the IPM model than for the conventional incremental change model. This matches empirical findings that once it reaches a stable state, trust tends to be "sticky" and difficult to change. People do not notice or react to every little event.

Third, the IPM model predicts that trust changes will have momentum: when a change in trust level occurs, subsequent changes trend in the same direction. In the incremental progression model, random events generate two similar changes in a row, on average. In the IPM-based models, the same stream of events generates between 4 and 8 consecutive changes in the same direction (Table 1, Trend column).

\section{Conclusion}

The IPM contributes by providing a process theory of trust change that is grounded in social psychology's cognitive information processing. First, the model addresses the mental mechanisms people use as they are confronted by trust-related events. The Information Processing Model suggests these mechanisms must each be engaged for trust to change. Second, the model illustrates several reasons trust does not change under many circumstances that one might think warrant change. The model indicates that trust may be "sticky" or resistant to change, but that change can and will occur. The model is especially applicable to studies of reputation, soft trust, social networking, and e-commerce/mobile commerce.

\section{References}

1. Blau, P.M.: Exchange and Power in Social Life. John Wiley \& Sons, New York (1964)

2. Holmes, J.G.: Trust and the Appraisal Process in Close Relationships. In: Jones, W.H., Perlman, D. (eds.) Advances in Personal Relationships (2), pp. 57-104. Jessica Kingsley, London (1991)

3. Jarvenpaa, S.L., Knoll, K., Leidner, D.E.: Is Anybody Out There? Antecedents of Trust in Global Virtual Teams. Journal of Management Information Systems 14, $29-64$ (1998)

4. Louis, M.R., Sutton, R.I.: Switching Cognitive Gears: From Habits of Mind to Active Thinking. Human Relations 44, 55-76 (1991)

5. Luhmann, N.: Trust and Power. John Wiley, New York (1979)

6. Marsh, S.P.: Formalising trust as a computational concept, Ph.D. Thesis, University of Stirling (1994)

7. Muir, B.M.: Trust in Automation: Part I. Theoretical Issues in the Study of Trust and Human Intervention in Automated Systems. Ergonomics 37, 1905-1922 (1994)

8. Murray, S.L., Holmes, J.G.: A Leap of Faith? Positive Illusions in Romantic Relationships. Personal and Social Psychology Bulletin 23, 586-604 (1997)

9. Newell, A.: Unified Theories of Cognition. Harvard University Press, Cambridge (1990) 